\title{
SIAC Filtering for Nonlinear Hyperbolic Equations
}

Xiaozhou Li and Jennifer K. Ryan

We present the results of the symmetric and one-sided Smoothness-Increasing Accuracy-Conserving (SIAC) filter applied to a discontinuous Galerkin (DG) approximation for two examples of nonlinear hyperbolic conservation laws. The traditional symmetric SIAC filter relies on having a translation invariant mesh, periodic boundary conditions and linear equations. However, for practical applications that are modelled by nonlinear hyperbolic equations, this is not feasible. Instead we must concentrate on a filter that allows error reduction for nonuniform/unstructured meshes and non-periodic boundary conditions for nonlinear hyperbolic equations. This proceedings is an introductory exploration into the feasibility of these requirements for efficient filtering of nonlinear equations.

\section{Introduction and Motivation}

In this article, we consider the usefulness of superconvergence extraction techniques for discontinuous Galerkin (DG) approximations to nonlinear hyperbolic equations of the form

$$
\begin{array}{r}
u_{t}+\sum_{i=1}^{d} f(u)_{x_{i}}=0,(\mathbf{x}, t) \in \Omega \times(0, T], \\
u(\mathbf{x}, 0)=u_{o}(\mathbf{x}), \mathbf{x} \in \Omega .
\end{array}
$$

The specific extraction technique that we consider is Smoothness-Increasing AccuracyConserving (SIAC) filtering. We consider this technique as it is known for reducing

Xiaozhou Li

Delft Institute of Applied Mathematics, Delft University of Technology, 2628CD Delft, Netherlands, e-mail: X.Li-2@tudelft.nl

Jennifer K. Ryan

School of Mathematics, University of East Anglia, Norwich NR4 7TJ, United Kingdome-mail: Jennifer.Ryan@uea.ac.uk 
the oscillations in the DG error as well as the error itself, while increasing the continuity of the numerical approximation.

Mathematically, the symmetric SIAC filter relies on having a translation invariant mesh, periodic boundary conditions and a linear equation. However, for practical applications that are modelled by nonlinear hyperbolic equations, this is not feasible. Instead we must concentrate on a filter that allows error reduction for nonuniform/unstructured meshes and non-periodic boundary conditions for nonlinear hyperbolic equations. The question we seek to answer is how feasible are these requirements for efficient filtering of nonlinear equations.

\section{Background}

\subsection{Discontinuous Galerkin Methods}

In this section, we merely summarize the important properties of the discontinuous Galerkin method that are useful in superconvergence extraction. More on these methods can be found in $[2,3]$.

The useful properties are:

- An approximation space that consists of piecewise polynomials of degree $\leq k$.

- Weak continuity at element interfaces.

- A variational formulation

$$
\left(\left(u_{h}\right)_{t}, \psi\right)_{\Omega}+\sum_{i=1}^{d}\left(-\left(f_{i}\left(u_{h}\right), \psi_{x_{i}}\right)_{\Omega}+\sum_{K} \int_{\partial K} \widehat{f}_{i}\left(u_{h}^{L}, u_{h}^{R}\right) v_{i} \psi d s\right)=0,
$$

where the summation is over all elements in our discretized domain.

These properties allow us to obtain the following error estimates for the DG solution for linear hyperbolic equations:

- $u-u_{h} \sim \mathscr{O}\left(h^{k+1}\right)$ in $L^{2}$ for sufficiently smooth initial data, $u_{0}$.

- $u-u_{h} \sim \mathscr{O}\left(h^{2 k+1}\right)$ in a negative order norm.

We emphasize that these estimates rely on having smooth enough initial data and a linear equation so that information propagates along characteristics. In the case of nonlinear hyperbolic equations, the initial data may be smooth enough, but characteristics may intersect, forming a shock.

\subsection{Smoothness-Increasing Accuracy-Conserving (SIAC) Filtering}

The Smoothness-Increasing Accuracy-Conserving (SIAC) filter is a form of superconvergence extraction that filters out oscillations in the error. It is performed by convolving the DG solution with a B-spline kernel at the final time,

$$
u_{h}^{\star}(x)=\left(K_{h}^{2(k+1), k+1} \star u_{h}(\cdot, T)\right)(x) .
$$


Using a SIAC filter on linear hyperbolic equations, one may show that

$$
\left\|u-K_{h}^{2(k+1), k+1} \star u_{h}\right\|_{0, \Omega_{0}} \leq C h^{2 k+1},
$$

for a translation invariant mesh. This is based upon the work $[1,6,8,4]$.

The symmetric convolution kernel is a central B-spline kernel given by

$$
K^{(r+1, \ell)}(x)=\sum_{\gamma=0}^{r} c_{\gamma}^{(r+1, \ell)} \psi^{(\ell)}\left(x-x_{\gamma}\right),
$$

where $K_{H}^{(r+1, \ell)}(x)=\frac{1}{H} K^{(r+1, \ell)}\left(\frac{x}{H}\right), x_{\gamma}=-\frac{r}{2}+\gamma$ and generally $r=2 k$ and $\ell=k+1$. We note that $\psi^{(\ell)}$ is a central $\mathrm{B}$-spline of order $\ell$, and $H$ is generally the translation invariance of the mesh. The weighting coefficients of the B-splines are given by the linear system

$$
K^{(r+1, \ell)} \star p=p, \quad p=1, x^{2}, \ldots, x^{r} .
$$

Note that convolving the DG solution with such a kernel produces an approximation that is a polynomial of degree $r+1 \leq 2 k+1$ with continuity of $\ell-2 \leq k-1$. Further note that the post-processing stencil width is of length $(r+\ell) H$.

\subsection{Boundary Filtering}

The kernel given in Equation (6) is for post-processing smooth regions, away from boundaries. However, when near a boundary or discontinuity, this needs to be sufficiently modified to balance accuracy constraints with error reduction and computational efficiency. It has recently been shown [7] that a suitable modification is given by

$$
K^{(r+1, \ell)}(x)=\underbrace{\sum_{\gamma=0}^{r} c_{\gamma}^{(\ell)} \psi^{(\ell)}\left(x-x_{\gamma}-\lambda(x)\right)}_{\text {Shifted filter }}+\underbrace{c_{r+1}^{(\ell)}(x-(\bar{x}-1))^{\ell-1} \chi_{[\bar{x}-1, \bar{x}]}}_{\text {Special B-spline }} .
$$

This kernel uses $r+1$ central B-splines that are shifted to accommodate a nonsymmetric support near a boundary or discontinuity along with a general B-spline that aids in improving computational efficiency and reducing errors in regions where one-sided filters are necessary.

At the price of computational efficiency and error reduction we have had to give up the property of superconvergence for $\ell-1 \geq 2$. In the interior, we achieve superconvergence of order $r+1 \leq 2 k+1$, but still only have convergence of order $\ell-1 \leq k+1$ at the boundaries. However, in the case of linear approximations and $k=1$, we still achieve a global superconvergence order of three, even in the boundary regions. This clearly shifts our focus to error reduction and hence allows us to more closely examine how the SIAC filter could aid in error reduction for nonlinear equations whose solution contains a discontinuity. 


\section{SIAC Filtering for Nonlinear Hyperbolic Equations}

There has been previous work in SIAC filtering for nonlinear hyperbolic equations. However, the work was restricted to nonlinear equations with a smooth solution. In [5], the following theorem was given:

Theorem 1. Assume we have a smooth solution to Equation (1) whose DG approximate is given by $u_{h}$. If $\left|f_{i}^{\prime \prime}\right| \leq M$, then

$$
\left\|\left(u-u_{h}\right)(T)\right\|_{-(k+1), \Omega} \leq C h^{2 k+m},
$$

where $m=0, \frac{1}{2}, 1$, depends on the numerical flux and $k>\frac{d}{2}$.

As a consequences of this higher order convergence in the negative-order norm, we then have $\mathscr{O}\left(h^{2 k+m}\right)$ convergence of the post-processed solution in the $L^{2}$-norm.

\section{Numerical examples}

Although the theory has been established for smooth solutions, it is interesting to investigate the application of the SIAC filter to nonsmooth solutions. To demonstrate the possibilities of the SIAC filtered solution for such solutions, we present two examples: First, a one-dimensional Burgers equation after the shock has developed; and lastly, the double Mach reflection problem of the two-dimensional Euler equations.

The steps of the filtering process are as follows:

- Calculate the DG approximation to the equation at the final time $t=T$.

- Identify "troubled cells", i.e., where there is a discontinuity.

- Calculate the SIAC filtered solution

- Use a symmetric filter in smooth regions, a distance of at least $\frac{r+\ell}{2} h$ away from boundaries or discontinuities.

- In "troubled cell regions", use a boundary filter.

\subsection{One-dimensional Burgers Equation}

For the first example, we consider the equation

$$
u_{t}+u u_{x}=0, \quad u(x, 0)=\sin (x), \quad x \in[0,2 \pi], \quad T=1 .
$$

Note that this equation contains a shock at $x=\pi$. We have implemented the symmetric filter in smooth regions and the boundary filter in the elements next to the boundaries and shocks. No filter is implemented in the element that contains the shock. The results for the errors are presented in Table 1 and Figure 1. 
Table 1 The $L^{2}$-error of the DG solution and the SIAC filtered DG Solution for $\mathbb{P}^{2}$ and $\mathbb{P}^{3}$ using the boundary filter in the appropriate regions. Errors are calculated away from the shock.

\begin{tabular}{|c||cc||cc||cc||cc|}
\hline \multicolumn{1}{|c||}{} & \multicolumn{1}{c||}{ DG } & \multicolumn{2}{c||}{ SIAC DG } & \multicolumn{2}{c||}{ DG } & \multicolumn{2}{c|}{ SIAC DG } \\
\hline Mesh & $L^{2}$ error order & $L^{2}$ error order & $L^{2}$ error order & $L^{2}$ error order \\
\hline \multicolumn{1}{|||}{} & \multicolumn{4}{c|}{$\mathbb{P}^{2}$} & \multicolumn{4}{c|}{$\mathbb{P}^{3}$} \\
\hline 40 & $1.02 \mathrm{E}-05$ & - & $5.28 \mathrm{E}-06$ & - & $4.89 \mathrm{E}-08$ & - & $5.18 \mathrm{E}-08$ & - \\
\hline 60 & $3.29 \mathrm{E}-06$ & 2.80 & $1.61 \mathrm{E}-06$ & 2.94 & $1.07 \mathrm{E}-08$ & 3.74 & $1.59 \mathrm{E}-09$ & 8.60 \\
\hline 80 & $1.46 \mathrm{E}-06$ & 2.83 & $6.94 \mathrm{E}-07$ & 2.92 & $3.08 \mathrm{E}-09$ & 4.33 & $1.61 \mathrm{E}-10$ & 7.96 \\
\hline 100 & $7.84 \mathrm{E}-07$ & 2.78 & $3.63 \mathrm{E}-07$ & 2.90 & $1.33 \mathrm{E}-09$ & 3.76 & $3.40 \mathrm{E}-11$ & 6.96 \\
\hline
\end{tabular}

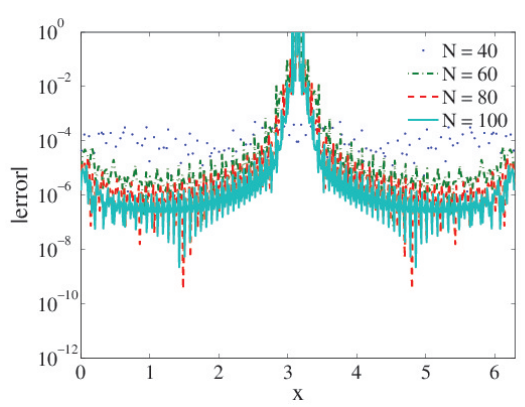

(a) DG Error

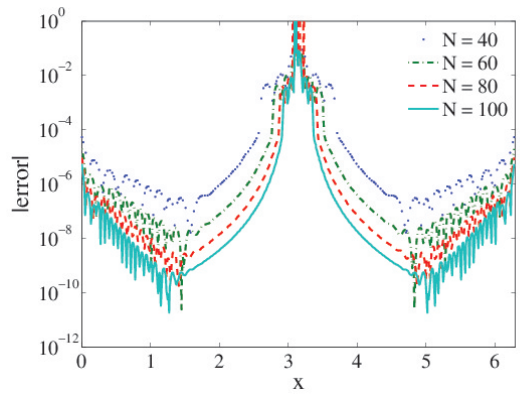

(b) SIAC DG Error

Fig. 1 Plots of pointwise errors of the DG solution and the SIAC filtered DG Solution for $\mathbb{P}^{2}$ using the boundary filter in the appropriate regions.

\subsection{Two-dimensional double Mach reflection}

In this example we apply the SIAC filter, including the boundary filter, to the two dimensional Euler equations for the double Mach reflection problem. We use the multiwavelet troubled cell indicator of Vuik [9] and plot the results for a zoomedin region of the solution in Figure 2. Note that from the results given for Burgers equation we expect that the difference when we examine the two solutions will be small. However, we do observe some reduced oscillations with the SIAC Filtered DG approximation.

\section{Conclusions and Future Work}

SIAC filtering holds promise in applications to nonlinear equations, although their exact usefulness remains unclear. Traditionally, SIAC filtering uses B-splines to induce smoothness on the DG field and enhance accuracy. This traditionally allows order improvement from $\mathscr{O}\left(h^{k+1}\right)$ to $\mathscr{O}\left(h^{2 k+m}\right)$ for smooth regions. At the boundaries, order is reduced for improved computational efficiency. For nonlinear equations, their usefulness depends on the boundedness of the flux function and the chosen 
numerical flux. From our observations, the filtering appears to reduce oscillations in regions where applied. How exactly it should be applied is the subject of on-going research.

Acknowledgements Portions of this research are supported by the European Office of Aerospace Research and Development under grant numbers FA8655-09-1-3055 and FA8655-13-1-3017. We would like to thank Liangyue Ji (Minnesota) for useful discussions and Thea Vuik (TU Delft) for providing the data used in the double Mach reflection example.

\section{References}

1. J.H. Bramble and A.H. Schatz, Higher order local accuracy by averaging in the finite element method, Mathematics of Computation, 31, pp. 94-111 (1977).

2. B. Cockburn, Discontinuous Galerkin methods for convection-dominated problems, HighOrder Methods for Computational Physics, vol. 9 of Lecture Notes in Computational Science and Enginerring, Springer, 1999.

3. B. Cockburn and C.-W. Shu, Runge-Kutta Discontinuous Galerkin methods for convectiondominated problems, Journal of Scientific Computing, 16, pp. 173-261 (2001).

4. B. Cockburn, M. Luskin, C.-W. Shu, and E. Süli, Enhanced accuracy by post-processing for finite element methods for hyperbolic equations, Mathematics of Computation, 72, pp.577606 (2003).

5. L. Ji, Y. Xu and J.K. Ryan, Negative-order norm estimates for nonlinear hyperbolic conservation laws, Journal of Scientific Computing, 54, 269-310 (2013).

6. M.S. Mock and P.D. Lax, The computation of discontinuous solutions of linear hyperbolic equations, Communications on Pure and Applied Mathematics, 18, pp. 423-430 (1978).

7. X. Li, R.M. Kirby, J.K. Ryan, C. Vuik, Computationally Efficient Position-Dependent Smoothness-Increasing Accuracy-Conserving (SIAC) filtering: The uniform mesh case, preprint (2013).

8. V. Thomée, High order local approximations to derivatives in the finite element method, Mathematics of Computation, 31, pp. 652-660 (1977).

9. M.J. Vuik, Limiting and shock detection for discontinuous Galerkin solutions using multiwavelets, TU Delft MSc Thesis, 2012-08-24.

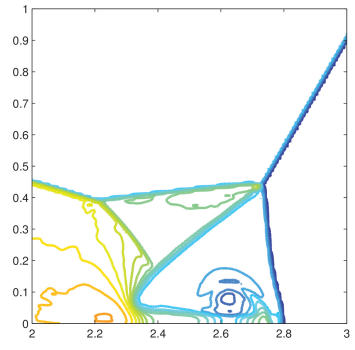

(a) DG Approximation

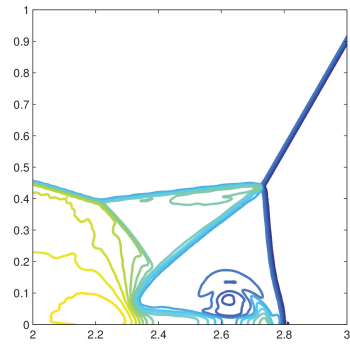

(b) SIAC Filtered DG

Fig. 2 Results for the DG approximation and SIAC Filtered DG approximation when applied to the double Mach reflection problem. 